wie möglich befinden, so müssen die Schwankungen bei ungleicher Neigung des Arbeitstisches sehr unbedeutend sein. Wern man nun den Apparat so stellt, dass die Durchschnittsfläche der Röhren $\boldsymbol{M}_{1}$ und $\boldsymbol{M}_{2}$ mit dem Kiel des Schiffes parallel ist, so dürften die Bewegungen, welche das Schwanken des Fahrzeugs verursachen könnte, vollständig ausgeglichen sein.

Eine gute Vorstellung von der Genauigkeit der hier beschriebenen Methode kann man durch die Beobachtungen, welche in den folgenden Aufsätzen erwähnt werden, erhalten. Nach diesen dürfte der Mittelfehler für Stickstoffgas- und Kohlensäurebestimmungen kaum $\pm 0,2$ Proc. von der bestimmten Quantität übersteigen. Bei der Arbeit an Bord muss man natürlich den Fehler etwas grösser annehmehmen, doch dürfte dieser Apparat in allen Fällen, hinsichtlich des Stickstoffgases und der Kohlensăure, sichere Ergebnisse als die früher angewandten Methoden geben können. Was aber das Sauerstoffgas betrifft, so dürfte das Verhältniss umgekehrt sein.

Bunsen ) hat gezeigt, dass sauerstoffhaltiges Wasser, welches mit Quecksilber in Berührung steht, immer die Entstehung von etwas Quecksilberoxydul verursacht. Die Menge desselben ist zwar immer klein, doch hinreichend, um den Sauerstoffgehalt des Wassers um einige Procente zu vermindern. $\mathrm{Da}$ in dem hier beschriehenen Apparate das Wasser, welches untersucht werden soll, stets mit dem Quecksilber in Berührung ist, so kann derselbe hinsichtlich der Sauerstoffmenge kein zuverlässiges Resultat geben.

Um das Verhältniss zwischen den absorbirten Stickstoffund Sauerstoffmengen zu untersuchen, dürfte man Jacobsen's Methode mit Erfolg anwenden können. Zu diesem Zweck ist die absolute Menge der ausgekochten Luft von geringer Bedeutung.

\title{
III. Ueber das Stickstoffgas des Meerwassers.
}

Lange hat der Irrthum geherrscht, dass der Luftgehalt in den tieferen Theilen des Meeres ungeheuer gross sei und

1) Gasometrische Methoden. Braunschweig 1877. S. 221. 
442 Hamberg: Beiträge zur Chemie des Meerwassers.

einigermassen dem enormen Druck entspreche. Aber für eine solche Annahme haben alle neueren Untersuchungen auf diesem Gebiete keine Stütze gebracht.

Der Stickstoffgehalt in einem Tiefwasser ist derselbe, den dieses Wasser, als es sich zuletzt an der Oberfäche befand, absorbirt hatte. Dagegen ist die Menge des Sauerstoffes durch oxydirbare organische Stoffe und die lebenden Thiere stets etwas verringert. Die Menge des Stickstoffgases, welche ein Meerwasser absorbirt, hängt hauptsächlich von der Temperatur ab, weil die kleinen Schwankungen des Druckes von weniger Bedeutung sind. Kennt man daher den Absorptionscoëfficienten des Stickstoffgases für das Meerwasser und ausserdem den Stickstoffgehalt in einem Tiefwasser, so kann man mit einiger. Sicherheit die Temperatur berechnen, die dieses Wasser hatte, als es sich das letzte Mal an der Oberfiäche befand. Mit Anwendung dieses Satzes, gelangte Tornö $\mathrm{e}^{\mathbf{y}}$ zu höchst interessanten Resultaten hinsichtlich der Circulation in dem norwegischen Nordmeere.

Der mit der Temperatur wechselnde Absorptionscoëfficient des Stickstoffgases im Meerwasser ist sowohl von Tornö $\mathrm{e}^{2}$ ) als auch von Dittmar ${ }^{3}$ ) untersucht worden, doch ist die Uebereinstimmung zwischen ihren Resultaten keineswegs gut. Bunsen $n^{4}$ und Dittmar haben den entsprechenden Coëfficienten in reinem $W$ asser bestimmt, und die Uebereinstimmung ihrer Angaben ist noch schlechter. Dass die Ungleichheiten wirklich bedeutend sind, ersieht man daraus, dass, wo Tornöe 14,40 gefunden, Dittmar 15,60, und wo Bunsen 20,35, Dittmar 24,40 gefunden hat.

Da ich während der Expedition des Freih. von Nordenskiöld 1883 ein kleines Material von Bestimmungen des Stickstoffgases im Meerwasser gesammelt hatte, musste ich

1) The norwegian North-Atlantic Expedition 1876-1878. Chemi ete. by Hercules Tornöc S. 72.

2) Das. S. 17.

3) Raport on the Scientific Results of the Voyage of H. M. S. Challenger etc. London 1884. Vol, I. S. 160.

4) Gasometrische Methoden, S. 192-209. 
Hamberg: Beiträge zur Chemie des Meerwassers. 443

zu deren Verwerthung einige Normalbestimmungen haben, um sie damit zu vergleichen. Je mehr ich in die Kritik von Bunsen's, Dittmar's und Tornöe's Bestimmungen hineinkam, desto mehr wurde ich überzengt, dass diese ganze Frage einer Revision bedürfe. Unter anderem hielt ich es für nothwendig, nicht nur die Wirkung der Temperatur, sondern auch die des Salzgehaltes auf die Menge des Stickstoffgases zu untersuchen.

Die Absorptionsversuche, welche weiter unten erwähnt werden, sind hauptsächlich nach einer neuen Bestimmungsmethode gemacht worden, indem der oben beschriebene Apparat angewandt wurde. Die Versuche wurden mit Wasser von den Salzgehalten 0 Proc., 1,7784 Proc., 2,6580 Proc. und 3,5126 Proc. und bei den Temperaturen $0^{\circ},+10^{\circ}$ und $+20^{\circ}$ vorgenommen.

Die Wasserprobe, welche untersucht werden sollte, befand sich während der Absorption selbst in dem Aussiedeballon $B$, und dieser war in ein ziemlich grosses, gut isolirtes Wasserbad, dessen Temperatur constant gehalten wurde, niedergesenkt. Die angewandte Luft wurde aus dem Freien genommen und durch sauergemachtes Wasser, Baumwolle und eine Kugelröhre geleitet, die Wasser von dernselben Salzgehalt und derselben Temperatur, wie das Absorptionswasser, enthielt. Zuletzt wurde diese Luft in feinen Bläschen durch den Aussiedeballon ( $B)$ geleitet, in welchem die Flüssigkeit, die der Gegenstand der Untersuchung war, sich befand. Das Durchsaugen der Luft wurde durch eine Wasserluftpumpe bewirkt. Der obere Theil des Aussiedeballons stand mit einem offenen Quecksilbermanometer in Verbindung. Es dürfte unnöthig sein hinzuzufügen, dass das Barometer bei jeder Absorption beobachtet, und dass der Druckunterschied, welchen das Manometer angab, von dem abgelesenen Barometerstande subtrahirt wurde.

Das Aussieden wurde in Uebereinstimmung mit dem, was in der Beschreibung über den Apparat erzählt ist, vorgenommen. Bei der Analyse des ausgekochten Gases wurde zuerst die Kohlensäure mit Kalilauge, darauf das Sauerstoffgas mit pyrogallussaurem Kali absorbirt. Dieses $A b$ - 
444 Hamberg: Beiträge zur Chemie des Meerwassers. sorptionsmittel würde eine Stickstoffgasmenge ungefähr um 0,2 Proc. zu hoch geben, welcher Fehler ohne Berücksichtigung gelassen werden könnte, besonders da wohl immer ein kleiner Rest von Gas in dem ausgekochten Wasser zurückbleibt.

In der untenstehenden Tabelle sind die Resultate sämmtlicher Absorptionen vereinigt. Die abgelesenen Gasvolume sind bis auf $0^{\circ}$ und $760 \mathrm{Mm}$. und zur Absorption bei $760 \mathrm{Mm}$. trockenem Luttdruck reducirt.

\begin{tabular}{|c|c|c|c|c|c|c|c|c|}
\hline Nr. & $\begin{array}{c}\text { Bezeich- } \\
\text { nung } \\
\text { des } \\
\text { Wassers }\end{array}$ & $\begin{array}{c}\mathrm{Cl} \\
\text { in Grm. } \\
\text { per Lit. }\end{array}$ & $\begin{array}{c}\text { Spec. } \\
\text { Gewicht } \\
\text { bei } \frac{0^{0}}{4^{0}} \\
\text { (berechn.) }\end{array}$ & Proc. C] & $\begin{array}{l}\text { Proc. } \\
\text { Salz }\end{array}$ & $\begin{array}{l}\text { Temp. } \\
\text { (Cels.) }\end{array}$ & $\begin{array}{l}\text { Cubik- } \\
\text { Centim. } \\
\text { Stick- } \\
\text { stoffgas } \\
\text { per Lit. }\end{array}$ & $\begin{array}{c}\text { Dauer } \\
\text { der } \\
\text { Absorp } \\
\text { tion } \\
\text { (Stund. }\end{array}$ \\
\hline 1 & $a$ & 0,000 & 0,99987 & 0,0000 & 0,0000 & $0^{\circ}$ & 19,177 & 5 \\
\hline 2 & $"$ & $"$ & $"$ & $"$ & $"$ & $"$ & 19,101 & 16 \\
\hline 3 & $"$ & $"$ & $"$ & $"$ & $"$ & $+10^{\circ}$ & 15,145 & 4 \\
\hline 4 & $"$ & $"$ & $\eta$ & $"$ & " & $"$ & 15,142 & 4 \\
\hline 5 & $"$ & $"$ & $"$ & $n$ & $"$ & $+20^{\circ}$ & 12,639 & $4^{1 / 2}$ \\
\hline 6 & $n$ & $"$ & $n$ & $"$ & $"$ & $"$ & 12,617 & 6 \\
\hline 7 & b) & 9,998 & 1,01436 & 0,9831 & 1,7784 & $0^{\circ}$ & 15,685 & 16 \\
\hline 8 & $"$ & $"$ & $"$ & $"$ & $"$ & $"$ & 16,697 & $121 / 2$ \\
\hline 9 & $"$ & $"$ & $"$ & $"$ & $"$ & $"$ & 16,758 & 7 \\
\hline 10 & $"$ & $"$ & $"$ & $"$ & $"$ & $+10^{0}$ & 13,459 & $6^{1:}$ \\
\hline 11 & $"$ & $"$ & $"$ & $"$ & $n$ & $n$ & 13,448 & 5 \\
\hline 12 & $"$ & $"$ & $"$ & " & $"$ & $+20^{\circ}$ & 11,339 & 6 \\
\hline 13 & $"$ & $"$ & $"$ &, & " & $"$ & 11,299 & $4^{1 / 2}$ \\
\hline 14 & $c$ & 15,007 & 1,02139 & 1,4693 & 2,6580 & $0^{0}$ & 15,732 & 16 \\
\hline 15 & $"$ & $"$ & $"$ & $"$ & $\eta$ & $+10^{\circ}$ & 12,729 & $41 / 2$ \\
\hline 16 & $"$ & $"$ & $"$ & $"$ & ", & $+20^{\circ}$ & 10,750 & 3 \\
\hline 17 & $d$ & 19,965 & 1,02815 & 1,9418 & 3,5126 & $0^{0}$ & 14,856 & 8 \\
\hline 18 & $"$ & $"$ & $"$ & $"$ & $"$ & $"$ & 14,889 & $14^{t / 2}$ \\
\hline 19 & $"$ & , & $"$ & $"$ & $"$ & $"$ & 14,793 & 13 \\
\hline 20 & $"$ & " & $"$ & $"$ & $"$ & $"$ & 14,776 & $16^{1 / 2}$ \\
\hline 21 & $"$ & $"$ & $"$ & $n$ & $"$ & $n$ & 14,827 & $10 \%$ \\
\hline 22 & $"$ & $"$ & $"$ & $n$ & $"$ & " & 14,893 & 7 \\
\hline 23 & $"$ & $"$ & $"$ & $"$ & $"$ & $"$ & 14,865 & $14 \frac{1}{2}$ \\
\hline 24 & $"$ & $"$ & $\because$ & ", & $"$ & $"$ & 14,848 & 17 \\
\hline 25 & $"$ & $"$ & " & $"$ & " & , & 14,792 & 5 \\
\hline 26 & $"$ & $"$ & ", & $"$ & " & $+10^{\circ}$ & 12,058 & 5 \\
\hline 27 & $"$ & " & $"$ & $"$ & $"$ & $n$ & 12,060 & $51 / 2$ \\
\hline 28 & $"$ & $"$ & $"$ & $"$ & $"$ & $"$ & 12,019 & $4^{1 / 2}$ \\
\hline 29 & $"$ & $"$ & $"$ & $"$ & $"$ & $+20^{\circ}$ & 10,304 & 4 \\
\hline 30 & $"$ & $"$ & " & $"$ & $"$ & " & 10,165 & $61 / 2$ \\
\hline
\end{tabular}


Hamberg: Beiträge zur Chemie des Meerwassers. 445

Insofern die angewandte Methode mit keinen grösseren constanten Fehlern behaftet ist, dürfte obige Tabelle eine ziemlich wahre Vorstellung von der grösseren oder geringeren Zuverlässigkeit der Experimente geben können. Der Durchschnittsfehler für jede einfache Bestimmung beläuft sich bis auf $\pm 0,0276 \mathrm{Ccm}$. oder ungefähr 0,2 Proc. von der bestimmten Quantität.

In der untenstehenden Tabelle sind die Durchschnittszahlen für Wasser mit demselben Salzgehalt und von derselben Temperatur auf eine anschaulichere Weise aufgestellt.

\begin{tabular}{|c|c|c|c|c|c|c|c|}
\hline & $\begin{array}{c}\text { Bezeich- } \\
\text { nung }\end{array}$ & $\begin{array}{l}\text { Proc. } \\
\text { Salz }\end{array}$ & $\begin{array}{c}\text { Cubik- } \\
\text { Centim. } \\
\text { Stickstoff- } \\
\text { gas per Lit. } \\
\text { bei } 0^{0}\end{array}$ & Differ. & $\begin{array}{c}\text { Cubik- } \\
\text { Centim. } \\
\text { Stickstoff- } \\
\text { gas per Lit. } \\
\text { bei }+10^{\circ}\end{array}$ & Differ. & $\begin{array}{c}\text { Cubik- } \\
\text { Centim. } \\
\text { Stickstoff- } \\
\text { gas per Lit. } \\
\text { bei }+20^{\circ} \\
\end{array}$ \\
\hline & $a$ & 0,0000 & 19,139 & 3,995 & 15,144 & $2, \overline{516}$ & 12,628 \\
\hline \multirow[t]{2}{*}{ 芦 } & & 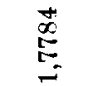 & $\begin{array}{l}\stackrel{\mathscr{H}}{\Im} \\
\text { oi }\end{array}$ & & 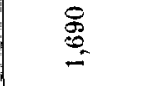 & & 总 \\
\hline & $b$ & 1,7784 & 16,713 & 3,259 & 13,454 & 2,135 & 11,319 \\
\hline \multirow[t]{2}{*}{ 它 } & & \begin{tabular}{l}
$\stackrel{0}{\sigma}$ \\
\multirow{2}{\infty}{} \\
0 \\
0
\end{tabular} & $\begin{array}{l}\vec{\infty} \\
\sigma \\
\sigma\end{array}$ & & 恖 & & 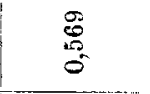 \\
\hline & $c$ & 2,6580 & 15,732 & 3,003 & 12,729 & 1,979 & 10,750 \\
\hline \multirow[t]{2}{*}{ 㟔 } & & 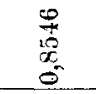 & $\begin{array}{l}\stackrel{x}{\tilde{x}} \\
\dot{x} \\
\sigma\end{array}$ & & $\begin{array}{l}0 \\
\infty \\
0 \\
0 \\
0\end{array}$ & & $\begin{array}{l}100 \\
0 \\
0\end{array}$ \\
\hline & $d$ & 3,5126 & 14,839 & 2,793 & 12,046 & 1,811 & 10,235 \\
\hline
\end{tabular}

Aus Obigem ergiebt sich, welchen grossen Einfiuss nicht nur die Temperatur, sondern auch der Salzgehalt auf die Stickstoffabsorption hat. Der Salzgehalt des Meeres ist zwar wenig variabel, aber auch diese kleine Variation ist nicht zu vergessen. Ein Unterschied von 0,2 in dem Salzprocent entspricht einem Unterschied von ungetähr 0,15 bis $0,2 \mathrm{Ccm}$. in dem Stickstoffgehalt.

Die Variation der Stickstoffmenge mit dem Salzgehalt bei der Temperatur $0^{\circ},+10^{\circ}$ und $+20^{\circ}$ kann durch folgende Interpolationsformeln ausgedrückt werden : 
446 Hamberg: Beiträge zur Chemie des Meerwassers.

\begin{tabular}{r|c|c}
\hline Bei & $\begin{array}{l}\text { absorbirt Wasser vom Salzgehalt } s \text { Proc. bei } \\
\text { trockenem Luftdruck aus atmosphärischer Luft die } \\
\text { Stickstoffmenge }\end{array}$ & $\begin{array}{c}\text { Formel } \\
\text { Nr. }\end{array}$ \\
\hline \hline $0^{\circ}$ & $19,139-1,5078 . s+0,080762 . s^{2}$ & 1 \\
$+10^{0}$ & $15,144-1,0204 . s+0,039412 . s^{2}$ & 2 \\
$+20^{\circ}$ & $12,628-0,79254 . s+0,031681 . s^{2}$ & 3
\end{tabular}

Beim Ausrechnen dieser Formeln habe ich die Bestimmungen für das Wasser $c$ ausgenommen, welche nicht sowie die übrigen aus mehreren einfachen Beobachtungen berechnete Durchschnittzahlen sind. Für dieses Wasser (Salzgehalt $=2,658$ Proc.) geben die Formeln folgende Stickstoffmengen:

$\begin{array}{ccc}\text { Temp. } & \text { Berechnete } & \text { Beobachtete } \\ 0^{\circ} & \text { Stickstoffmenge } & \text { Stickstoffmenge } \\ +10^{\circ} & 15,702 & 15,732 \\ +20^{\circ} & 12,710 & 12,729 \\ & 10,745 & 10,750\end{array}$

Ebenso wie die oben stehenden Formeln fuir die $\mathbf{A b}$ hängigkeit der Stickstoffmenge von dem Salzgehalt, können natürlich auch Formeln für die Wirkung der Temperatur berechnet werden. Eine solche, direct aus den Beobachtungen ausgesuchte Formel für destillirtes Wasser, würde folgendes Aussehen haben:

$$
N=19,139-0,4735 t+0,007+t^{2} .
$$

Diese Formel ist für Temperaturen, welche nur unbedeutend höher als $+20^{\circ}$ sind, völlig unbrauchbar, weil das dritte Glied allzu schnell wächst. Für eine Temperatur von beinahe $+40^{\circ}$ giebt die Formel einen mit der Temperatur steigenden Stickstoffgehalt an. Aber nimmt man an, dass die Stickstoffmenge bei $100^{\circ}=0 \mathrm{Ccm}$. ist, und berechnet eine neue Formel mit einem vierten Glied, so erhält man

$$
N=19,139-0,48616 t+0,009301 t^{2}-0,00006353 t^{3},
$$

welche für Temperaturen über $+20^{\circ}$ ziemlich wahrscheinliche Werthe giebt und für Temperaturen zwischen $0^{\circ}$ und $20^{\circ}$ Werthe, die mit denen, welche die erste Formel giebt, beinahe identisch sind. Mit Hülfe der Formeln Nr. 1, 2 und 3 sammt der obenerwähnten Annahme habe ich für 
Hamberg: Beiträge zur Chemie des Meerwassers. 447 die Salzgehalte $0,1,2,3$ und 3,5 Proc. die untenstehenden Formeln berechnet.

\begin{tabular}{c|c|c}
\hline $\begin{array}{c}\text { Wasser } \\
\text { vom } \\
\text { Salzgehalt }\end{array}$ & $\begin{array}{c}\text { absorbirt bei } 760 \mathrm{Mm} \text {. trockenem Luftdrucke } \\
\text { und der Tempcratur } t \text { aus atmospharischer Iuft } \\
\text { die Stickstoffmenge }\end{array}$ & $\begin{array}{c}\text { Formel } \\
\text { Nr. }\end{array}$ \\
\hline \hline 0,0 & $19,139-0,48616 t+0,009301 \quad t^{2}-0,00006353 t^{3}$ & 4 \\
1,0 & $17,712-0,42828 t+0,0078736 t^{2}-0,00005362 t^{3}$ & 5 \\
2,0 & $16,446-0,38260 t+0,0068794 t^{2}-0,00004698 t^{3}$ & 6 \\
3,0 & $15,342-0,34922 t+0,0063180 t^{2}-0,00004360 t^{3}$ & 7 \\
3,5 & $14,851-0,33720 t+0,006200 \quad t^{2}-0,00004313 t^{3}$ & 8
\end{tabular}

Diese Formeln sind der Tabelle, welche hier unten mitgetheilt wird, zu Grunde gelegt. Die Stickstoffmengen für Temperaturen unter $0^{0}$ habe ich durch das Aussuchen von den wahrscheinlichsten Differenzen erhalten.

\section{Tabelle}

zeigend, wie viele Cubikcentimeter Stickstoffgas (bis auf $0^{\circ}$ und $760 \mathrm{Mm}$. reducirt) ein Liter Wasser von unten stehenden Salzgehalten und Temperaturen bei $760 \mathrm{Mm}$. trockenem Luftdruck absorbiren kann.

\begin{tabular}{|c|c|c|c|c|c|c|c|c|c|c|}
\hline 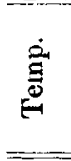 & 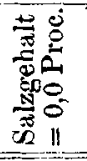 & 范 & 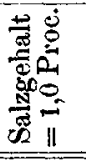 & 苞 & 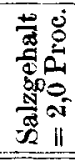 & 蛋 & 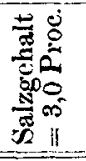 & 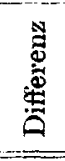 & 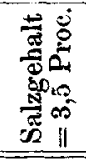 & 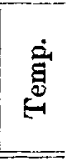 \\
\hline$-3^{0}$ & 20,69 & 1,63 & 19,06 & 1,40 & 17,66 & 1,21 & 16,45 & 0,52 & 15,93 & $-3^{\circ}$ \\
\hline--2 & 20,15 & 1,55 & 18,60 & 1,36 & 17,24 & 1,17 & 16,07 & 0,52 & 15,55 & -2 \\
\hline-1 & 19,64 & 1,49 & 18,15 & 1,31 & 16,84 & 1,14 & 15,70 & 0,51 & 15,19 & -1 \\
\hline 0 & 19,14 & 1,43 & 17,71 & 1,26 & 16,45 & 1,11 & 15,34 & 0,49 & 14,85 & 0 \\
\hline+1 & 18,66 & 1,37 & 17,29 & 1,22 & 16,07 & 1,07 & 15,00 & 0,48 & 14,52 & +1 \\
\hline 2 & 18,20 & 1,31 & 16,89 & 1,18 & 15,71 & 1,04 & 14,67 & 0,47 & 14,20 & 2 \\
\hline 3 & 17,76 & 1,26 & 16,50 & 1,14 & 15,36 & 1,01 & 14,35 & 0,46 & 13,89 & 3 \\
\hline 4 & 17,34 & 1,22 & 16,12 & 1,10 & 15,02 & 0,98 & 14,04 & 0,44 & 13,60 & 4 \\
\hline$\check{5}$ & 16,93 & 1,17 & 15,76 & 1,06 & 14,70 & 0,95 & 13,75 & 0,43 & 13,32 & 5 \\
\hline 6 & 16,54 & 1,13 & 15,41 & 1,02 & 14,39 & 0,92 & 13,47 & 0,43 & 13,04 & 6 \\
\hline 7 & 16,17 & 1,09 & 15,08 & 0,99 & 14,09 & 0,90 & 13,19 & 0,41 & 12,78 & 7 \\
\hline 8 & 15,81 & 1,05 & 14,76 & 0,96 & 13,80 & 0,87 & 12,93 & 0,40 & 12,53 & 8 \\
\hline 9 & 15,47 & 1,01 & 14,46 & 0,93 & 13,53 & 0,85 & 12,68 & 0,39 & 12,29 & 9 \\
\hline 10 & 15,14 & 0,98 & 14,16 & 0,90 & 13,26 & 0,82 & 12,44 & 0,38 & 12,06 & 10 \\
\hline 11 & 14,83 & 0,95 & 13,88 & 0,87 & 13,01 & 0,80 & 12,21 & 0,38 & 11,83 & 11 \\
\hline 12 & 14,53 & 0,92 & 13,61 & 0,85 & 12,76 & 0,77 & 11,99 & 0,37 & 11,62 & 12 \\
\hline 13 & 14,25 & 0,89 & 13,36 & 0,83 & 12,53 & 0,76 & 11,77 & 0,35 & 11,42 & 13 \\
\hline 14 & 13,98 & 0,87 & 33,11 & 0,80 & 12,31 & 0,74 & 11,57 & 0,34 & 11,23 & 14 \\
\hline
\end{tabular}


448 Hamberg: Beiträge zur Chemie des Meerwassers.

\begin{tabular}{|c|c|c|c|c|c|c|c|c|c|c|}
\hline 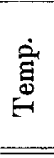 & 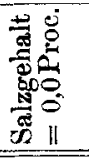 & 㺼 & 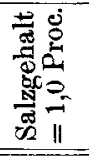 & 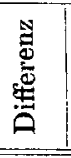 & 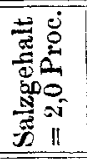 & 荀 & 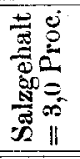 & 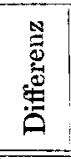 & 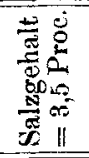 & $\stackrel{\dot{\theta}}{\vec{y}}$ \\
\hline 15 & 13,73 & 0,85 & 12,88 & 0,78 & 12,10 & 0,72 & 11,38 & 0,34 & 11,04 & 15 \\
\hline 16 & 13,48 & 0,82 & 12,66 & 0,77 & 11,89 & 0,70 & 11,19 & 0,32 & 10,87 & 16 \\
\hline 17 & 13,25 & 0,81 & 12,44 & 0,74 & 11,70 & 0,68 & 11,02 & 0,32 & 10,70 & 17 \\
\hline 18 & 13,03 & 0,79 & 12,24 & 0,73 & 11,51 & 0,66 & 10,85 & 0,31 & 10,54 & 18 \\
\hline 19 & 12,82 & 0,77 & 12,05 & 0,71 & 11,34 & 0,65 & 10,69 & 0,30 & 10,39 & 19 \\
\hline 20 & 12,63 & 0,76 & 11,87 & 0,70 & 11,17 & 0,63 & 10,54 & 0,29 & 10,25 & 20 \\
\hline 21 & 12,44 & 0.75 & 11,69 & 0,68 & 11,01 & 0,62 & 10,39 & 0,28 & 10,11 & 21 \\
\hline 22 & 12,27 & 0,74 & 11,53 & 0,67 & 10,86 & 0,61 & 10,25 & 0,27 & 9,98 & 22 \\
\hline 23 & 12,10 & 0,73 & 11,37 & 0,65 & 10,72 & 0,60 & 10,12 & 0,27 & 9,85 & 23 \\
\hline 24 & 11,95 & 0,72 & 11,23 & 0,65 & 10,58 & 0,58 & 10,00 & 0,27 & 9,73 & 24 \\
\hline 25 & 11,81 & 0,72 & 11,09 & 0,64 & 10,45 & 0,57 & 9,88 & 0,26 & 9,62 & 25 \\
\hline
\end{tabular}

Auf der Tafel $\mathrm{V}$ findet sich obenstehende Tabelle graphisch dargestellt, welches mit Vortheil angewandt werden kann, wenn es sich um Stickstoffmengen, Temperaturen und Salzgehalte, die zwischen den in der Tabelle aufgenommenen liegen, handelt. Um die Interpolation zwischen den gezeichneten Curven zu erleichtern, giebt es eine „Scala“ für das Abnehmen der Stickstoffmenge mit zunehmendem Salzgehalt und constanter Temperatur, wie auch für das Abnehmen der Temperatur mit zunehmendem Salzgehalt und constanter Stickstoffmenge.

Wie schon erwähnt, war es im Anfang dieser Untersuchung mein Wunsch, nur Bunsen's, Tornöe's und Dittmar's widersprechende Angaben beurtheilen zu können. Um ihre Resultate mit den meinigen zu vergleichen, habe ich die folgende Tabelle aufgestellt.

Die Zahlen, welche ich für reines Wasser gefunden, habe ich, um den Vergleich mit denjenigen von Bunsen und Dittmar zu erleichtern, auf Absorption von reinem Stickstoffgas reducirt, indem ich sie mit 0,7905 dividirt habe. Der Stickstoffgehalt der angewandten Luft wurde zu 79,05 Volumenprocent angenommen. 
Hamberg: Beiträge zur Chemie des Meerwassers. 449

\begin{tabular}{|c|c|c|c|c|c|c|c|}
\hline \multirow{2}{*}{ 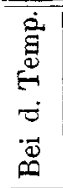 } & \multicolumn{3}{|c|}{$\begin{array}{l}\text { unter dem Drucke von } 760 \\
\text { reines Wasser aus reinem } \\
\text { Stickstoffgas untenstehende } \\
\text { Cubikcent. Stickstoff nach }\end{array}$} & \multicolumn{3}{|c|}{$\begin{array}{l}\text { Mm. absorbirt ein Liter } \\
\text { "Meerwasser" aus atmosphä- } \\
\text { rischer Luft untenstehende } \\
\text { Cubikcent. Stickstoff nach }\end{array}$} & \multirow[t]{2}{*}{ 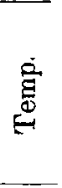 } \\
\hline & Bunsen & Dittmar & Hamberg & Tornöe & Dittmar & Hamberg & \\
\hline $0^{n}$ & 20,35 & 24,40 & 24,21 & 14,40 & 15,60 & 14,85 & $0^{\circ}$ \\
\hline+5 & 17,94 & 21,62 & 21,42 & 13,25 & 13,86 & 13,32 & +5 \\
\hline 10 & 16,07 & 19,43 & 19,15 & 12,10 & 12,47 & 12,06 & 10 \\
\hline 15 & 14,78 & 17,65 & 17,37 & 10,95 & 11,34 & 11,04 & 15 \\
\hline 20 & 14,03 & 16,19 & 15,98 & - & 10,41 & 10,25 & 20 \\
\hline 25 & - & 14,95 & 14,94 & - & 9,62 & 9,62 & 25 \\
\hline
\end{tabular}

Die Differenzen sind im Allgemeinen bei den niederen Temperaturen am grössten. Was reines Wasser betrifft, so dürfte die Tabelle die ziemlich gute Uebereinstimmung zwischen Dittmar's und meinen Bestimmungen darthun, während die von Bunsen gefundenen Absorptionscoëfficienten für Stickstoffgas allzu niedrig sind.

In Bezug auf das Meerwasser zeigen meine Bestimmungen dagegen eine viel bessere Uebereinstimmung mit denjenigen von Tornöe als deren Dittmar's. Deswegen will ich an Folgendes erinnern:

Dass Tornöe bei der niedrigsten Temperatur $\left(0^{\circ}\right)$ einen relativ sehr niedrigen Stickstoffgehalt gefunden, dürfte sich theils aus dem Grunde erklären lassen, dass er die Absorption nicht in demselben Gefäss vorgenommen, in welchem das Aussieden geschah, theils auch durch die von ihm angewandte Methode Jacobsen's. Der Druck, unter welchem die Flüssigkeit in Jacobsen's Apparat kocht, ist höher bei grösseren Mengen ausgekochten Gases, während die Wärmequelle ungefähr dieselbe ist. Deshalb kann man nicht erwarten, aus einer grösseren Menge absorbirten Gases im Vergleiche ebensoviel ausgetrieben zu erhalten, als aus einer kleineren. $\left.{ }^{1}\right)$

Weder Tornöe noch Dittmar haben genau angegeben,

1) Dass Tornöe einen zu niedrigen Stickstoffgehalt bei $0^{0}$ gefunden, dürfte zu dem von ihm ausgesprochenen unrichtigen Satze, dass die Stickstoffmenge der Temperatur proportional ist, Veranlassung gegeben haben.

Journal f. prakt. Chemie [2] Bd. 33. 
450 Hamberg: Beiträge zur Chemie des Meerwassers.

von welchem Salzgehalt das Wasser war, welches sie bei den Absorptionen anwandten. Tornöe sagt, dass er sich eines Meerwassers von einigermassen hohem specifischen Gewicht bedient habe. Dittmar dagegen hat ein sehr problematisches, von ihm selbst bereitetes Meerwasser angewandt. Er sagt jedoch, dass er sich Mühe gegeben, eine Salzlösung hervorzubringen, welche hinsichtlich ihrer $\mathrm{Zu}$ sammensetzung so nahe wie möglich dem Meerwasser komme.

Für $0^{0}$, die Temperatur, bei welcher die Unterschiede zwischen Tornöe's, Dittmar's und meinen Resultaten am grössten sind, ist die Zahl, welche ich gefunden, die Durchschnittzahl von neun einfachen, gut mit einander übereinstimmenden Bestimmungen. Dieselbe muss deshalb eine gewisse Glaubwürdigkeit haben, insofern die Methode, die ich angewandt, mit keinen constanten, mir unbekannten Fehlerquellen behaftet ist.

\section{Von der Kohlensänre im Meerwasser.}

Von allen Bestandtheilen des Meerwassers hat keiner sich so schwer mit Sicherheit bestimmen lassen, als die Kohlensäure. Ich glaube behaupten zu dürfen, dass man jetzt noch keinen richtigen Begriff von dem wahren Kohlensäuregehalt des Meeres oder von den Schwankungen desselben hat. Und ebenso unsicher wie man die Kenntniss von der Quantität der Kohlensäure ansehen kann, ebenso schwankend ist die Auffassung von den Bindemitteln, welche die Kohlensäure im Meerwasser zurückhalten. Ein kurzer Rückblick auf die Ansichten in Betreff dieser Frage, welche in letzterer Zeit aufgeworfen ist, dürfte daher nicht ohne Interesse sein.

Jacobsen ${ }^{1}$, der Chemiker der deutschen PommeraniaExpedition, pflegte, um die Kohlensäure im Meerwasser zu bestimmen, dasselbe bis zur Trockne zu destilliren und die Kohlensäure im Destillat zu bestimmen. Er fand in dem Wasser der Nordsee im Durchschnitt ungefähr $0,1 \mathrm{Grm} . \mathrm{CO}_{2}$ pro Liter, welche Angabe der Wahrheit einigermassen entspricht. Jacobsen meint, dass nur ein geringer Theil der

1) Ann. Chem. Pharm. 167, 1. 